\title{
Design of Bandpass Transversal Filters Employing a Novel Hybrid Structure
}

\author{
Mónica Martínez-Mendoza, Juan Sebastián Gómez-Díaz, David Cañete-Rebenaque, Student Member, IEEE, \\ Jose Luis Gómez-Tornero, Member, IEEE, and Alejandro Alvarez-Melcon, Senior Member, IEEE
}

\begin{abstract}
A novel structure for the implementation of compact transversal bandpass filters is proposed in this paper. This new proposal consists of a hybrid structure, based on the combination of two different technologies: the waveguide and microstrip. It is shown that the novel hybrid microwave filter is able to implement both a second- and third-order filtering function with up to two or three transmission zeros, respectively. In this way, a practical implementation of a fully canonical transversal filter with a third degree response is for the first time directly achieved. In addition, the way to control the positions of transmission zeros with the new technology is treated in this paper. It is shown that symmetric or asymmetric responses and also filtering functions with a complex pair of transmission zeros can be easily obtained. To demonstrate the validity of this novel structure, several implementation examples are presented. A prototype has been designed, manufactured, and successfully tested, showing the practical validity of the new structure.
\end{abstract}

Index Terms-Bandpass filters, coupling matrices, resonator filters, synthesis techniques, transmission zeros, transversal filters.

\section{INTRODUCTION}

$\mathbf{T}$ HE INTEREST in the implementation of transmission zeros at precise frequencies in microwave filters arose as a direct consequence of the need to reject unwanted signals. For this purpose, several techniques and different filter topologies have been developed in the last few decades. The introduction of cross-couplings between nonadjacent resonators in the coupling scheme of the filter has been the design method traditionally used to achieve this goal [1]. Nevertheless, in more recent contributions, alternative schemes for microwave resonator filters have also been proposed [2].

One of the most outstanding proposed topology was the transversal filter structure, whose coupling matrix can be directly synthesized using the technique presented in [3]. This structure differs from traditional ones in the fact that multiple input/output couplings are allowed. In addition, no coupling between resonators is introduced. Furthermore, fully canonical filtering functions may be synthesized if a direct coupling between the source and load is introduced. With this fully canonical transversal configuration, $N$ transmission zeros can

Manuscript received April 18, 2007; revised July 19, 2007 and August 8, 2007. This work was supported by the Centro de Investigación Científica y Tecnológica under Spanish National Project TEC2004-04313-C02-02/TCM and under Regional Seneca Project 02972/PI/05.

The authors are with the Telecommunications and Electromagnetics Group, Technical University of Cartagena, Cartagena E-30202, Spain (e-mail: shanaz00@hotmail.com; madhap@ono.com; david.canete@upct.es; josel.gomez@upct.es; alejandro.alvarez@upct.es).

Color versions of one or more of the figures in this paper are available online at http://ieeexplore.ieee.org.

Digital Object Identifier 10.1109/TMTT.2007.909613 be implemented with an $N$ th-degree filtering function for maximum selectivity.

Several practical implementations of transversal filters have been proposed in the last years. Different examples in printed and waveguide technology can be found in [4] and [5]. However, the practical implementation of fully transversal topologies is difficult when the order of the filter is high. This is because of the special routing scheme of transversal filters, where couplings from all the resonators to the input/output ports must be implemented, while at the same time inter-resonator couplings must be avoided. This difficulty has limited the practical implementation of transversal topologies to filters of second order [4]-[6]. When higher order filters are needed, rotations of the original $N+2$ transversal coupling matrix are applied to eliminate undesired couplings or to create new couplings between resonators [3], [7]. When the use of rotations is not possible to achieve a given desired topology, one can still resort to optimization techniques applied to the coupling matrix entries [8]. Higher order filters can also be designed by cascading several sections of second-order transversal filters [5], [9].

Following the fast development of transversal filters, a new filtering structure has been recently proposed in [10]. The special feature of the novel proposal is that a waveguide resonance is combined with a microstrip resonance to implement a second-order transversal filter; therefore, the given name of hybrid waveguide-microstrip technology. It was shown that the structure is able to implement a second-order transversal filter with direct coupling whose topology is known as the modified doublet [6]. This hybrid microwave filter combines, for the first time, the waveguide and microstrip technologies in a unique filter. The main advantage of the novel topology is that it exploits the benefits of both technologies, thus obtaining a very compact design.

This paper is focused on the further development of transversal microwave filters in hybrid waveguide-printed technology. New examples using the second-order structure are presented, showing the capability of the structure to control the position of the two transmission zeros. Besides, measured results of a second-order manufactured prototype are presented for validation.

Finally, using the hybrid technology concept, a novel implementation of a third-order fully canonical transversal filter is proposed. For the first time, it is shown that, using the hybrid technology, a very compact structure can be used for the implementation of third-order transversal filters. The third-order filter, which can be directly implemented with the new structure, follows the coupling scheme shown in Fig. 1.

Note that since this corresponds to a fully transversal topology, the direct synthesis technique presented in [3] can be used. Therefore, similarity transformations or optimization 


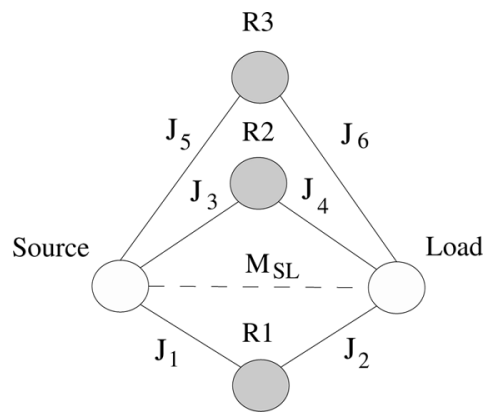

Fig. 1. Coupling scheme of a third-order fully canonical transversal filter. $J_{1}-J_{6}$ represent the couplings between source $S$, load $L$, and the resonators. $M_{S L}$ represents direct coupling from source to load.

TABLE I

COUPLING MATRIX OF THE MODIFIED DOUBLET. From [10]

\begin{tabular}{|c|cccc|}
\cline { 2 - 5 } \multicolumn{1}{c|}{} & $\mathrm{S}$ & 1 & 2 & $\mathrm{~L}$ \\
\hline $\mathrm{S}$ & 0 & $J_{1}$ & $J_{3}$ & $M_{S L}$ \\
1 & $J_{1}$ & $M_{11}$ & 0 & $J_{2}$ \\
2 & $J_{3}$ & 0 & $M_{22}$ & $J_{4}$ \\
$\mathrm{~L}$ & $M_{S L}$ & $J_{2}$ & $J_{4}$ & 0 \\
\hline
\end{tabular}

techniques are not needed. To the authors' knowledge, this is the first time that a practical implementation of a fully transversal filter of order 3 is proposed. In addition, this paper describes how the transmission zeros of the filter can be controlled using the different parts of the hybrid structure. In this way, both symmetric and asymmetric responses for maximum selectivity above and/or below the passband can be easily synthesized. We also show how pairs of transmission zeros can be placed in the complex plane to achieve a tradeoff between selectivity and group delay equalization. The behavior of the structures is illustrated with several implementation examples, highlighting the usefulness of the novel topology.

\section{DESIGN AND STRUCTURE DESCRIPTION}

The hybrid structure under study is able to implement either a second- or third-order filter. The necessary theory to understand the behavior of the second-order filter, which implements the so-called modified doublet, was detailed in [10]. In that study, the basic hybrid structure that implements the modified doublet was described. The coupling matrix $M$ of the second-order scheme is known to be of the form shown in Table I.

In the hybrid structure, the open line microstrip resonator in the printed circuit behaves as resonator $R_{1}$ of the modified doublet, whereas an longitudinal section magnetic (LSM) mode excited in the partially filled waveguide behaves as resonator $R_{2}$ (see [10]). This mode is TM with respect to the direction normal to the dielectric ( $z$-axis), and is also known as a hybrid LSM mode with respect to the $x$-axis when studying partially filled waveguides [11]. On the other hand, it is an established fact that for the modified doublet one of the four couplings must be negative. The change in sign of the electric field associated to the LSM mode, from the input to the output port, is responsible for this negative coupling, as recognized in [10]. However, it is possible to design the printed resonator to act as a simple half-wavelength open microstrip resonator since no change in sign is required for $R_{1}$.

The design procedure to implement a fixed filtering function using the hybrid structure can be carried out by following the idea of separating the design task into several simpler tasks, which was first introduced in [12]. In this way, the value of each dimension of the structure to implement the desired coupling terms can be obtained. First, we compute the $(N+2)$ transversal matrix associated with a fixed second-order filter. Next, using the coupling terms of the above $(N+2)$ coupling matrix, i.e., the impedance inverters $\left(J_{i}\right)$, the prototype de-normalization process (see, e.g., [13]) is applied. This process allows us to obtain the values of the required resonant frequencies of each resonator in asynchronously tuned filters $\left(f_{0, k} ; k=1,2\right)$. The de-normalization process also leads to the values of the required external quality factors $\left(Q_{e, k}\right)$ of each resonator. Once these values are known, the different resonators in the structure can be isolated to synthesize the required coupling elements. Specifically, we first look for the required frequency response of the resonant LSM mode. To do this, we eliminate the printed line microstrip resonator, and then we adjust the port lengths $L_{\text {in }}$ and $L_{\text {out }}$, and the waveguide width $b$ in order to achieve the required external quality factor and resonant frequency, respectively. Once the LSM resonance has been synthesized, the next step is to look for the required frequency response of the printed line microstrip resonator. Thereby we add the central microstrip line again and eliminate the presence of the other resonance. To do so, we detune it by setting the waveguide width to a larger value, while we adjust the microstrip line resonator. Now we can modify the line length $L_{r}$ and the coupling gaps $\omega_{1}$ and $\omega_{2}$ in order to obtain the required resonant frequency and external quality factor, respectively. Once the resonators have been individually synthesized, they are put together to verify that we obtain the desired filtering function inside the passband. However, the transmission zeros will probably not be located at the specified frequencies since the direct coupling term $M_{S L}$ still needs to be adjusted. To synthesize it, several iterations of the algorithm just described must be carried out, varying the dimensions $L_{2}$ and $L_{1}$ (the dielectric thickness and height of the cavity), until the transmission zeros are placed at the right locations. For the examples synthesized in this paper, we have observed that two or three iterations are usually enough to adjust the positions of the transmission zeros.

In the following, we propose to apply similar concepts for the synthesis of third-order fully canonical transversal filters using this hybrid waveguide-microstrip structure. The coupling routing of a third-order fully canonical transversal filter is sketched in Fig. 1. It consists of the input and output lines, which are coupled in a shunted configuration to three resonators at the same time. The hybrid structure used to implement this topology is shown in Figs. 2 (lateral view) and 3 (top view).

It can be seen that the basic waveguide structure is the same as in the modified doublet, and only the printed circuit is modified to introduce an additional line resonator. In the hybrid structure, the LSM mode of the partially filled waveguide behaves as resonator $R_{3}$ of the fully canonical transversal filter. In addition, the two microstrip resonators in the printed circuit behave as resonators $R_{1}$ and $R_{2}$ (see Fig. 3 ). Note that only two printed resonators are needed since the third additional resonance is due to the LSM mode excited in the partially filled cavity $\left(R_{3}\right)$.

The $(N+2)$ by $(N+2)$ coupling matrix of a third-order fully canonical transversal filter, calculated with the technique extensively presented in [3], is known to be of the form shown in Table II. In Table II, $J_{1}, J_{3}$, and $J_{5}$ represent the three input couplings, whereas $J_{2}, J_{4}$, and $J_{6}$ represent the output couplings. 


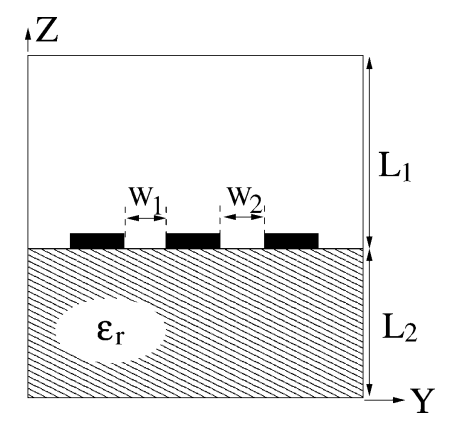

Fig. 2. Proposed hybrid waveguide-microstrip structure for a third-order transversal filter. Lateral view.

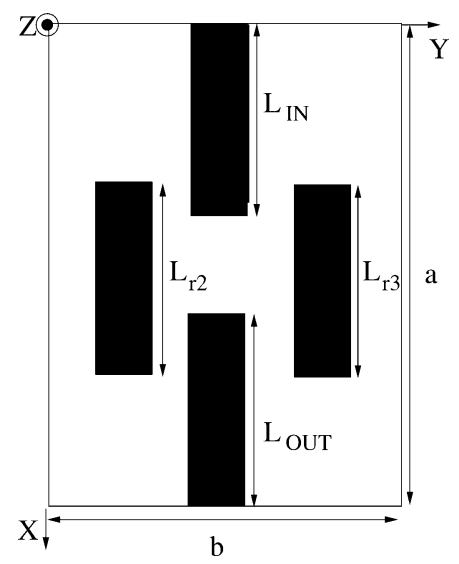

Fig. 3. Proposed hybrid waveguide-microstrip structure for the implementation of a third-order transversal filter. Top view. The width of the microstrip resonators. Input/output ports in the examples of this paper are $2 \mathrm{~mm}$.

TABLE II

COUPLING MATRIX OF A THIRD FULLY CANONICAL TRANSVERSAL FILTER

\begin{tabular}{|c|ccccc|}
\cline { 2 - 6 } \multicolumn{1}{c|}{} & $\mathrm{S}$ & 1 & 2 & 3 & $\mathrm{~L}$ \\
\hline $\mathrm{S}$ & 0 & $J_{1}$ & $J_{3}$ & $J_{5}$ & $M_{S L}$ \\
1 & $J_{1}$ & $M_{11}$ & 0 & 0 & $J_{2}$ \\
2 & $J_{3}$ & 0 & $M_{22}$ & 0 & $J_{4}$ \\
3 & $J_{5}$ & 0 & 0 & $M_{33}$ & $J_{6}$ \\
$\mathrm{~L}$ & $M_{S L}$ & $J_{2}$ & $J_{4}$ & $J_{6}$ & 0 \\
\hline
\end{tabular}

Furthermore, $M_{S L}$ represents direct source-load coupling. This term is important since it allows the implementation of a fully canonical filtering function. When this coupling exists, it is possible to implement $N$ transmission zeros for an $N$ th-order filter. Finally, the nonzero diagonal elements represent the self-couplings, i.e., the differences in the resonant frequencies of the three resonators, with respect to the center frequency of the filter. The diagonal elements are different from zero for asynchronously tuned filters, and this is a typical characteristic of transversal configurations.

As was discussed in [10], the $x$-component of the electric field of the excited LSM mode in the cavity couples to the input/ output ports. This component of the electric field is maximum at the input port, and it is zero at the center of the waveguide cavity. The field then changes sign at the output port. This change in sign makes it possible to synthesize the negative coupling required by the modified doublet implementation. In the thirdorder fully canonical transversal filter, the behavior is similar. At least one of the couplings in the matrix shown in Table II

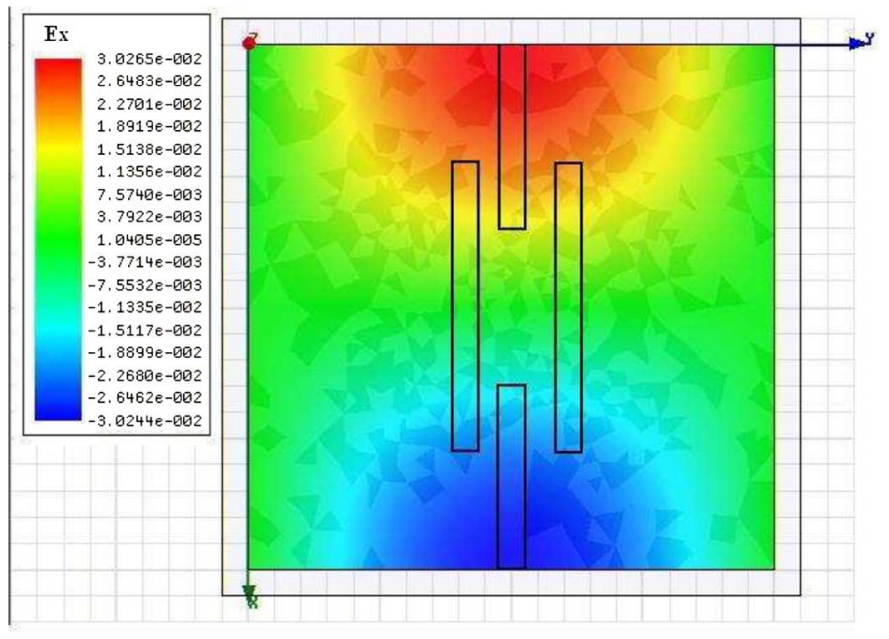

Fig. 4. Electric field $x$-component of the LSM mode inside the cavity, at the resonant frequency, for a third-order hybrid filter.

must be negative. The change in sign of the $x$-component of the electric field, associated with the excited LSM mode, is responsible for this negative coupling. The other two resonators are formed with half-wavelength printed lines so no further negative couplings are implemented. With this proposed configuration, therefore, only one negative coupling can be implemented in the matrix of Table II $\left(J_{5}=-J_{6}\right)$.

On the other hand, if the hybrid structure implements a transversal configuration, we must assure that the cross-coupling between both resonators $\left(R_{1}\right.$ and $\left.R_{2}\right)$ must be null. To explain why the coupling from the LSM mode and the printed line resonator can be neglected, we present in Fig. 4 the $x$-component of the field at the resonant frequency of the LSM mode. Due to the orientation of the printed lines, the $x$-component of the electric field will be the responsible for the coupling. We can observe in this figure that the printed resonator is placed at the center of the cavity, where the field has a zero. Consequently, the coupling from the LSM mode and the printed resonator will be small. On the contrary, the input/output printed lines are placed where the electric field is maximum and, therefore, stronger couplings can be obtained, as required by the transversal topology.

In addition, the different elements of this coupling matrix can be adjusted with the different geometrical parameters shown in Figs. 2 and 3. Specifically, the self-coupling $M_{33}$ is controlled by the lateral dimensions of the cavity ( $a$ and $b$ in Fig. 3) since they primarily control the resonant frequency of the excited LSM mode. The other two resonators are formed with printed microstrip lines. Therefore, the self-couplings $M_{11}$ and $M_{22}$ are controlled by their respective line lengths $\left(L_{r_{2}}\right.$ and $L_{r_{3}}$ in Fig. 3).

With regards to the values of the coupling elements out of the diagonal, the port lengths ( $L_{\mathrm{in}}$ and $L_{\mathrm{out}}$ of Fig. 3) will control the coupling between the input/output ports and the LSM mode (resonator $R_{3}$ ). Therefore, they control the $J_{5}$ and $J_{6}$ elements of the coupling matrix. The air gaps $w_{1}$ and $w_{2}$ (see Fig. 2) will further control the couplings between the input/output ports and the printed lines (resonators $R_{1}$ and $R_{2}$ ). Therefore, they can be used to appropriately adjust the elements $\left(J_{1}, J_{2}\right)$ and $\left(J_{3}, J_{4}\right)$ of the coupling matrix, respectively. 
As was established in [10], with the hybrid structure proposed, we will always have a direct coupling from the input to the output port (entry $M_{S L}$ in Table II). This indeed makes it possible to obtain fully canonical responses with a maximum of transmission zeros. This coupling is related to the excitation of the LSM mode, whose propagation is stopped by the presence of the lateral cavity walls. Accordingly, this paper will show that the direct coupling $\left(M_{S L}\right)$ can be controlled with the thickness of the dielectric substrate supporting the propagation of this LSM mode in the partially filled waveguide ( $L_{2}$ in Fig. 2). Using this concept, we will be able to show that there is full control in the position of the transmission zeros.

\section{RESUltS AND THEORETICAL DISCUSSION}

Here, several implementation examples are presented of second- and third-order transversal filters using the novel hybrid waveguide-microstrip technology. All the filters that will be discussed follow the coupling scheme of the modified doublet (see [10]) or the third-order coupling scheme shown in Fig. 1. The results predicted by the coupling matrix theory [3] will be compared with the results obtained from the electromagnetic analysis of the hybrid structures using the integral-equation technique computer-aided design (CAD) tool presented in [14]. For validation purposes, results obtained with a commercial CAD software tool called ADS will also be presented. Finally, measured results on a manufactured prototype are presented to show the practical validity of the new structure.

\section{A. Modified Doublet Implementation}

An asymmetric bandpass transversal filter with a secondorder response was designed in [10], showing excellent agreement between theoretical and full-wave simulations. The filter response exhibited two transmission zeros, one at each side of the passband, but they were placed asymmetrically with respect to the center frequency of the filter. Four new designs of a second-order transversal filter are included here to demonstrate the versatility of the new hybrid waveguide-microstrip structure.

The first example consist of a second-order bandpass filter with $-14 \mathrm{~dB}$ of return loss, and with all transmission zeros placed at infinity. The center frequency of the filter is $4.812 \mathrm{GHz}$ and the bandwidth is $90 \mathrm{MHz}$. Using the synthesis technique presented in [3], the coupling matrix for this filter is found to be

$$
M_{0}=\left(\begin{array}{cccc}
0 & 0.7000 & -0.7000 & 0 \\
0.7000 & -1.2085 & 0 & 0.7000 \\
-0.7000 & 0 & 1.2085 & 0.7000 \\
0 & 0.7000 & 0.7000 & 0
\end{array}\right)
$$

Note that the synthesis procedure leads to a matrix with no direct coupling between the source and load $\left(M_{S L}=0\right)$. This result is a direct consequence of the fundamental assumption made in [3] that a not fully canonical filter will always have zero direct coupling. However, in our hybrid structure, we will always have a direct coupling between input and output ports different from zero $\left(M_{S L} \neq 0\right)$. Therefore, the matrix $M_{0}$ cannot be directly implemented with our technology in the form shown above.

Nevertheless, by using the hybrid technology, we can implement a similar filter placing the transmission zeros in the complex plane. Since this filter corresponds to a fully canon- ical case, it will have a direct coupling different from zero. This can be verified by synthesizing a similar filter as before, but with a pair of transmission zeros located in the complex plane at $s= \pm 10.7+j \cdot 4.85 \cdot 10^{9}$. With the specified bandwidth and center frequency, the normalized transmission zeros are $s= \pm 10.7+j \cdot 0.8411$. The $(N+2)$ by $(N+2)$ coupling matrix obtained for this design is found to be

$$
M_{1}=\left(\begin{array}{cccc}
0 & -0.6975 & 0.7097 & 0.0102 \\
-0.6975 & -1.2095 & 0 & 0.6975 \\
0.7097 & 0 & 1.2019 & 0.7097 \\
0.0102 & 0.6975 & 0.7097 & 0
\end{array}\right) .
$$

Since the direct coupling is not zero $\left(M_{S L} \neq 0\right)$, this transfer function can be directly implemented with the hybrid technology proposed.

Note that, in the original matrix $M_{0}$, the resonator changing sign from input to output is tuned below the center frequency of the filter $\left(M_{22}\right.$ is positive and $\left.J_{3}=-J_{4}\right)$. Moreover, the resonator that does not change sign is tuned above the center frequency of the filter $\left(M_{11}\right.$ is negative and $\left.J_{1}=J_{2}\right)$. The opposite situation is applicable for the matrix $M_{1}$. This matrix corresponds to a filter with a pair of transmission zeros in the complex plane placed at $s= \pm 10.7+j \cdot 0.8411$. For this filter, the resonator changing sign from input to output is tuned above the center frequency of the filter $\left(M_{11}\right.$ is negative and $J_{1}=-J_{2}$ ). In the hybrid structure, the resonator changing sign is the LSM mode. Therefore, to implement a transfer function with two complex zeros, we must take the precaution of tuning the LSM mode above the center frequency of the filter. Finally, the line resonator must be tuned below the center frequency.

The response of the matrix $M_{1}$, as compared to the original matrix $M_{0}$, is shown in Fig. 5. It is interesting to observe that no transmission zeros at real frequencies are present in the response of the matrix $M_{1}$. However, the response can be synthesized with the novel hybrid waveguide-microstrip technology proposed in this paper since it has the direct coupling different from zero $\left(M_{S L} \neq 0\right)$. The final dimensions of the structure after the design procedure (according to [10, Figs. 2 and 3]) are shown in Table III.

The response of the designed hybrid structure agrees with the predicted behavior of the $M_{1}$ coupling matrix, as shown in Fig. 6. The results obtained with the ADS software tool are also included for validation.

In Fig. 5, we observe that the main effect when the transmission zeros are placed in the complex plane is a reduction in the far out-of-band rejection of the filter. It is known that this reduction in the rejection comes with an improvement in the group-delay flatness. This fact can be used to make designs with a tradeoff between selectivity and group-delay equalization.

To demonstrate this last point, Fig. 7 shows the maximum variation of the group delay within the passband as a function of the real part of the complex pair of transmission zeros. We observe that the maximum variation of the group delay is reduced by approximately $0.1 \mathrm{~ns}$ with respect to the initial Chebyshev filter $\left(M_{S L}=0\right)$. However, with this improvement in the group delay comes a reduction in the out-of-band rejection levels of the filter, as shown in Fig. 5.

Furthermore, it is interesting to point out that the achieved group-delay equalization in this example is practically the maximum equalization possible using this configuration. In the example, the complex pair of transmission zeros have a real part 


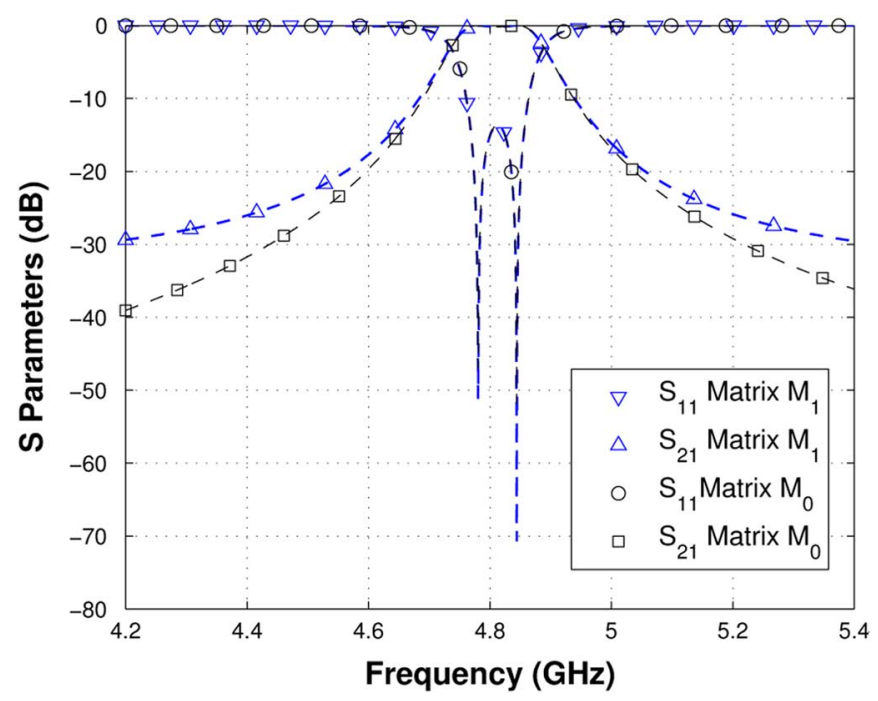

Fig. 5. Response of the matrix $M_{1}$, as compared to the original matrix $M_{0}$.

TABLE III

DimENSIONS OF THE SECOND-DEgREE FiLTER With A COMPLEX PAIR OF TRANSMISSION ZEROS PLACED AT $s= \pm 10.7+j \cdot 0.8411$

\begin{tabular}{c|c}
\hline Dimension & $\begin{array}{c}\text { Value } \\
\text { (in millimeters) }\end{array}$ \\
\hline \hline$a$ & 40 \\
$b$ & 37 \\
$L_{1}$ & 3 \\
$L_{2}$ & 2.14 \\
$L_{\text {in }}=L_{\text {out }}$ & 14 \\
$L_{r}$ & 23.9 \\
$w_{1}=w_{2}$ & 1.7 \\
$\varepsilon_{r}$ & 2 \\
\hline
\end{tabular}

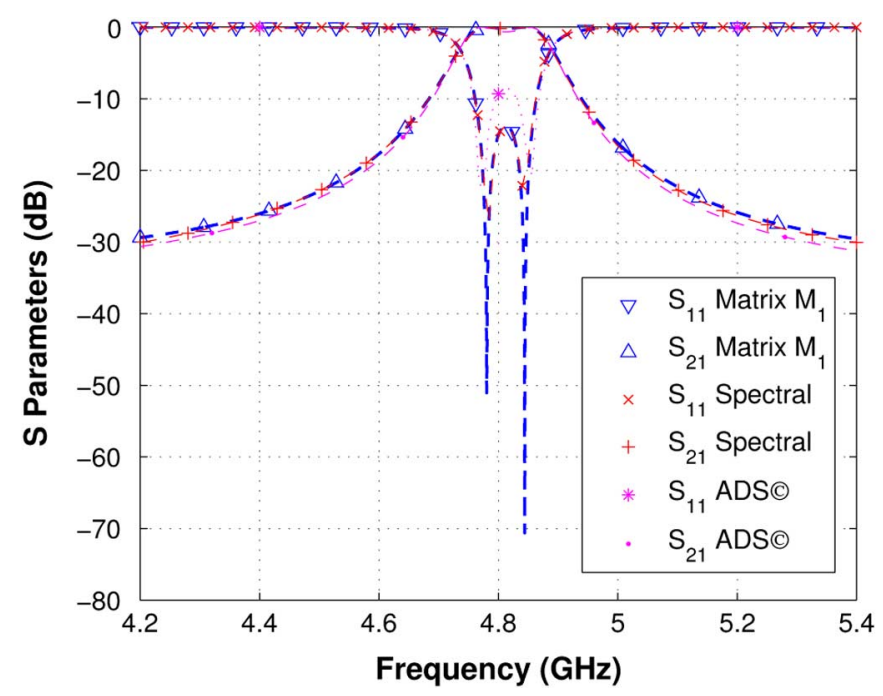

Fig. 6. Ideal model $\left(M_{1}\right)$ and simulated results for the bandpass transversal filter with a complex pair of transmission zeros placed at $s= \pm 10.7+j$. 0.8411 .

$(\delta= \pm 10.7)$ requiring a direct coupling term $M_{S L}=0.0102$. We have verified that the maximum direct coupling that can be implemented with this topology is approximately $M_{S L}=0.01$. However, the complex zeros can be placed farther from the

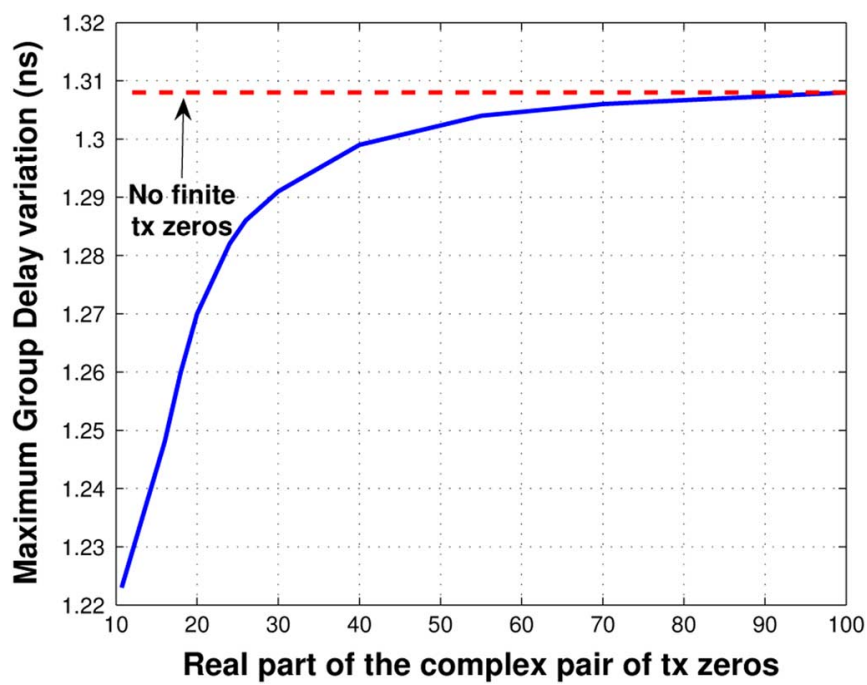

Fig 7. Maximum group delay variation within the passband versus the real part of the complex pair of transmission zeros (blue line in online version). The maximum variation of the group delay for the Chebyshev filter is also shown for reference (red line in online version).

imaginary axis by reducing this direct coupling with the adjustment of the heights $L_{1}$ and $L_{2}$ (see Fig. 2).

On the other hand, two transmission zeros placed on both sides of the passband can be implemented with the hybrid technology if the LSM mode resonance and the printed line resonator are interchanged with respect to the previous design. For this new design, the LSM mode resonance will be tuned below the center frequency of the filter and the printed line resonator will be tuned above. Since the filter is fully canonical in this case, the synthesis technique presented in [3] will automatically provide the direct input/output coupling $\left(M_{S L}\right)$ required to implement a desired transfer function.

Following this strategy, in the second example we designed a filter with two finite transmission zeros, one on each side of the passband $\left(f_{1}=4.224 \mathrm{GHz}\right.$ and $\left.f_{2}=5.106 \mathrm{GHz}\right)$. The filter has $-19 \mathrm{~dB}$ of return loss, a center frequency of $4.7 \mathrm{GHz}$, and $69 \mathrm{MHz}$ of bandwidth. Thereby, the normalized transmission zeros are $s=-j \cdot 17.3403$ and $s=j \cdot 12.5189$. The $(N+2)$ by $(N+2)$ coupling matrix obtained with the theory presented in [3] results in

$$
M_{2}=\left(\begin{array}{cccc}
0 & 0.8346 & -0.8658 & 0.0110 \\
0.8346 & -1.6303 & 0 & 0.8346 \\
-0.8658 & 0 & 1.6204 & 0.8658 \\
0.0110 & 0.8346 & 0.8658 & 0
\end{array}\right)
$$

We observe from the matrix that the resonator changing the sign of the coupling is tuned below the center frequency of the filter ( $M_{22}$ is positive and $J_{3}=-J_{4}$ ). In the hybrid structure, this resonator is implemented with the LSM mode. The matrix $M_{2}$ also indicates that the other resonator is tuned above the center frequency of the filter $\left(M_{11}\right.$ is negative and $\left.J_{1}=J_{2}\right)$. In the hybrid structure, this is implemented with the printed line resonator. Since the degree of the filter is equal to the number of finite transmission zeros (fully canonical), the direct input/ output coupling $\left(M_{S L}\right)$ is not zero.

The response of this filter is similar to the one presented in [10]. The main difference is that the transmission zeros are placed almost symmetrically with respect to the passband of the 


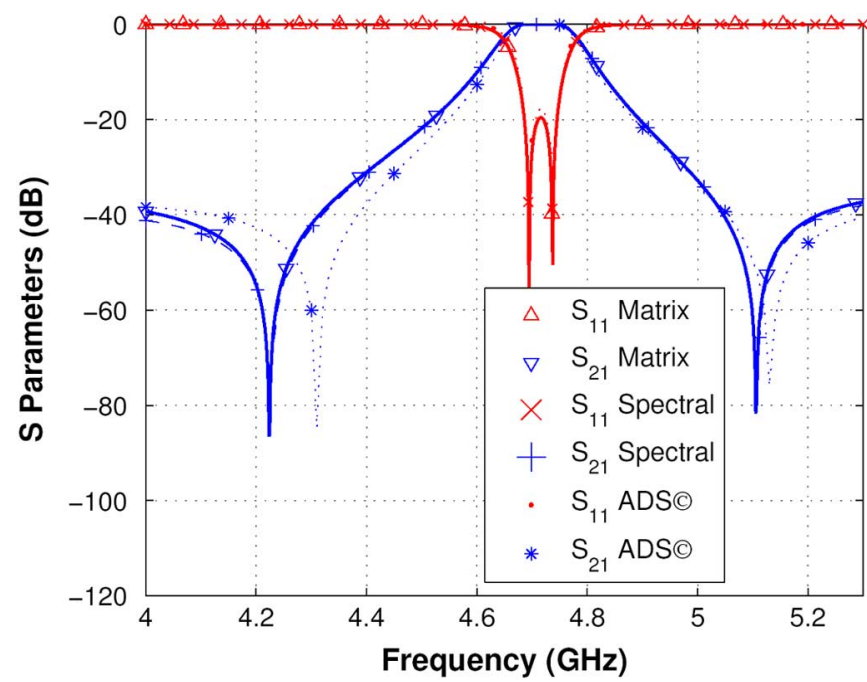

Fig. 8. Ideal model and simulated results for the bandpass transversal filter with symmetric response.

filter. This is shown in Fig. 8, where we show the response of the matrix $M_{2}$, together with the results of the designed hybrid structure.

By adjusting the different couplings, the hybrid structure is able to control the positions of the transmission zeros. In particular, this filter has a direct input/output coupling smaller than in the example presented in [10]. To reduce this coupling, the thickness of the dielectric has been reduced from $L_{1}=3 \mathrm{~mm}$ in the design of [10] to only $L_{1}=2.4 \mathrm{~mm}$ for the symmetric design. All the dimensions of the designed filter are collected in Table IV. In Fig. 8, we see very good agreement between the response of the designed hybrid structure and the results predicted by the $(N+2)$ coupling matrix. Results obtained with the commercial software ADS are also included for validation.

The next challenge is the synthesis of a filter with two transmission zeros on one side of the passband. This can be easily accomplished with the novel hybrid structure proposed by virtue of the zero shifting property [2]. For the third design example, the transmission zeros are located at $f_{1}=3.884 \mathrm{GHz}$ and $f_{2}=4.451 \mathrm{GHz}$. The filter is centered at $4.643 \mathrm{GHz}$ with a bandwidth of $57 \mathrm{MHz}$, thereby the normalized transmission zeros are $s=-j \cdot 29.2337$ and $s=-j \cdot 6.8821$. The return loss of the filter is $-18.5 \mathrm{~dB}$. The direct synthesis procedure of [3] leads to the following coupling matrix:

$$
M_{3}=\left(\begin{array}{cccc}
0 & 0.7150 & -0.9419 & 0.0104 \\
0.7150 & 1.5547 & 0 & 0.7150 \\
-0.9419 & 0 & -1.4566 & 0.9419 \\
0.0104 & 0.7150 & 0.9419 & 0
\end{array}\right)
$$

Note that the direct coupling $M_{S L}$ is also not zero in this case since this is a fully canonical design. The zero shifting property has also once again interchanged the two resonators, as compared to matrix $M_{2}$. Therefore, the resonance that changes sign must be tuned above the center frequency of the filter (the LSM mode), while the other resonance must be tuned below (the printed line resonator). This is the same situation as in the first example (see matrix $M_{1}$ above). The coupling matrix $M_{3}$ can be easily synthesized with the novel hybrid topology by tuning the LSM mode above the center frequency of the filter and the printed line resonator below.
TABLE IV

Dimensions of THE SECOND-DegReE FILTER WITH SYMMETRIC CHARACTERISTICS

\begin{tabular}{c|c}
\hline Dimension & $\begin{array}{c}\text { Value } \\
\text { (in millimeters) }\end{array}$ \\
\hline \hline$a=b$ & 40 \\
$L_{1}$ & 3 \\
$L_{2}$ & 2.4 \\
$L_{\text {in }}=L_{\text {out }}$ & 14 \\
$L_{r}$ & 23.74 \\
$w_{1}=w_{2}$ & 1.7 \\
$\varepsilon_{r}$ & 2 \\
\hline
\end{tabular}

TABLE V

DIMENSIONS OF THE SECOND-DEGREE FILTER WITH Two TRANSMISSION ZEROS BELOW THE PASSBAND

\begin{tabular}{c|c}
\hline Dimension & $\begin{array}{c}\text { Value } \\
\text { (in millimeters) }\end{array}$ \\
\hline \hline$a=b$ & 40 \\
$L_{1}$ & 3.2 \\
$L_{2}$ & 2.55 \\
$L_{\text {in }}=L_{\text {out }}$ & 15 \\
$L_{r}$ & 24.79 \\
$w_{1}=w_{2}$ & 2.37 \\
$\varepsilon_{r}$ & 2 \\
\hline
\end{tabular}

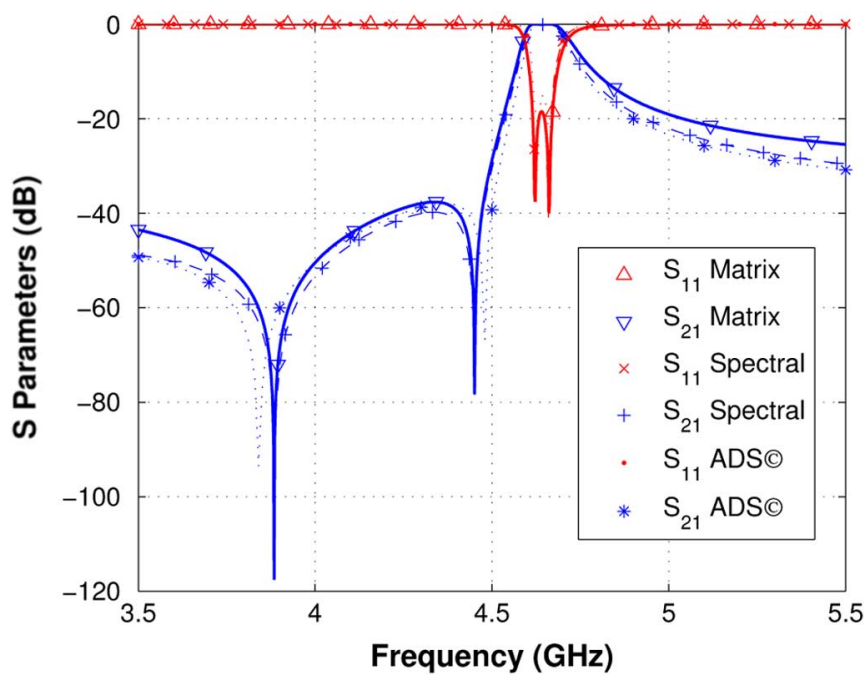

Fig. 9. Ideal model and simulated results for the bandpass transversal filter with two transmission zeros below the passband.

The dimensions of the structure are collected in Table V. The response of this structure is presented in Fig. 9, showing the two transmission zeros below the passband. We can observe that the electrical response of the designed hybrid structure agrees very well with the predicted behavior of the $M_{3}$ coupling matrix. Again, results obtained with the ADS software tool are included for validation.

Finally, a design with two transmission zeros on both sides of the passband has been manufactured and tested in order to provide experimental verification of the new structure. The substrate selected for manufacturing is an RT Duroid with relative permittivity $\varepsilon_{r}=2.2$ and thickness $1.57 \mathrm{~mm}$. The prototype implements a transfer function with two transmission zeros placed at $f_{1}=4.125 \mathrm{GHz}$ and $f_{2}=5.146 \mathrm{GHz}$. The filter is centered at $4.5 \mathrm{GHz}$ with a bandwidth of $136 \mathrm{MHz}$. With these data, the normalized transmission zeros are $s=-j \cdot 5.9260$ and $s=j \cdot 8.7724$. The return loss of the filter is $-15 \mathrm{~dB}$. The 
TABLE VI

Dimensions of THE MANUFACTURED SECOND-DEgREE FILTER With Two Transmission ZERos on Both SidEs OF THE PASSBAND

\begin{tabular}{c|c}
\hline Dimension & $\begin{array}{c}\text { Value } \\
\text { (in millimeters) }\end{array}$ \\
\hline \hline$a=b$ & 40 \\
$L_{1}$ & 2.62 \\
$L_{2}$ & 3.14 \\
$L_{\text {in }}=L_{\text {out }}$ & 13.5 \\
$L_{r}$ & 24 \\
$w_{1}=w_{2}$ & 1.4 \\
$\varepsilon_{r}$ & 2.2 \\
\hline
\end{tabular}

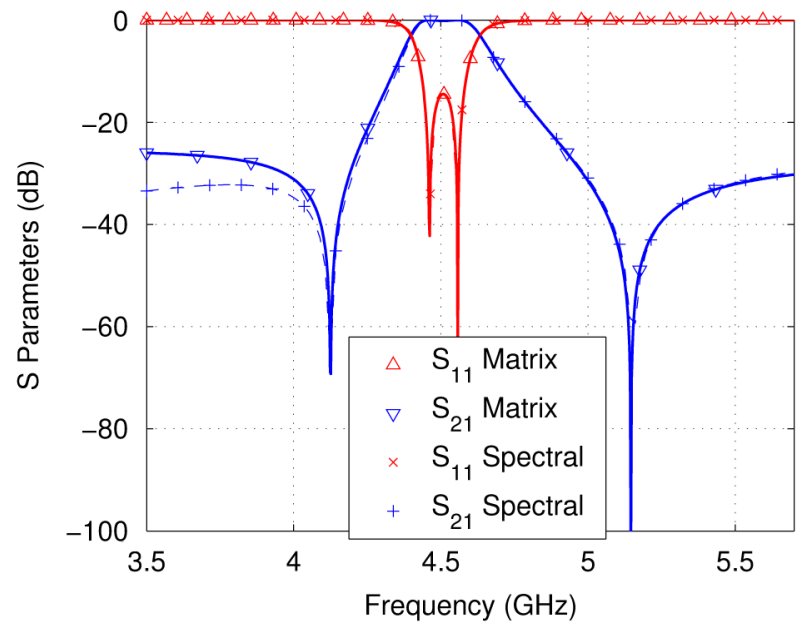

Fig. 10. Comparison of ideal model $\left(M_{4}\right)$ and lossless simulation results.

$(N+2)$ by $(N+2)$ coupling matrix obtained with the theory presented in [3] is

$$
M_{4}=\left(\begin{array}{cccc}
0 & -0.6852 & 0.7364 & 0.0253 \\
-0.6852 & 1.2723 & 0 & 0.6852 \\
0.7364 & 0 & -1.2483 & 0.7364 \\
0.0253 & 0.6852 & 0.7364 & 0
\end{array}\right) .
$$

The dimensions of the designed and manufactured filter are shown in Table VI. We can observe that the dielectric thickness is $L_{2}=3.14 \mathrm{~mm}$. In the manufactured prototype, this is achieved by piling up two RT Duroid substrates of $1.57-\mathrm{mm}$ thickness. In Fig. 10, we present the results obtained from the analysis of the hybrid structure, and we compare them with the predicted response of the $M_{4}$ matrix. Once more, good agreement is observed.

In addition, we present in Fig. 11 the measured results of the manufactured prototype, and we compare them with simulated results. In the simulations, losses are included in the dielectric substrate $(\tan \delta=0.004)$ and in the printed metallizations $(\sigma=$ $3 \cdot 10^{7} \Omega^{-1} / \mathrm{m}$ ). A photograph of the manufactured breadboard is shown in Fig. 12.

Results reveal that the minimum insertion loss of the filter inside the passband is $-1.15 \mathrm{~dB}$. We can also observe a slope in the insertion loss response of the filter. The insertion losses take a maximum value within the passband of $-2.48 \mathrm{~dB}$ at the frequency of $4.46 \mathrm{GHz}$. At the frequency of $4.59 \mathrm{GHz}$, the insertion loss is minimum $(-1.15 \mathrm{~dB})$. The slope in the insertion loss response of the filter reveals that a resonator with a higher quality factor has been combined with a resonator of

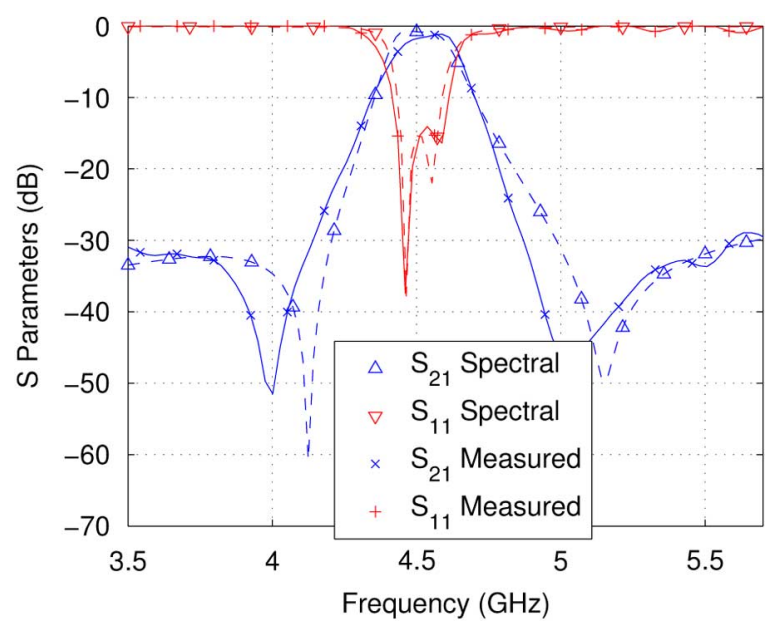

Fig. 11. Comparison between simulation results with losses $(\tan \delta=$ $0.004, \sigma=3 \cdot 10^{7} \Omega^{-1} / \mathrm{m}$ ) and measured results.

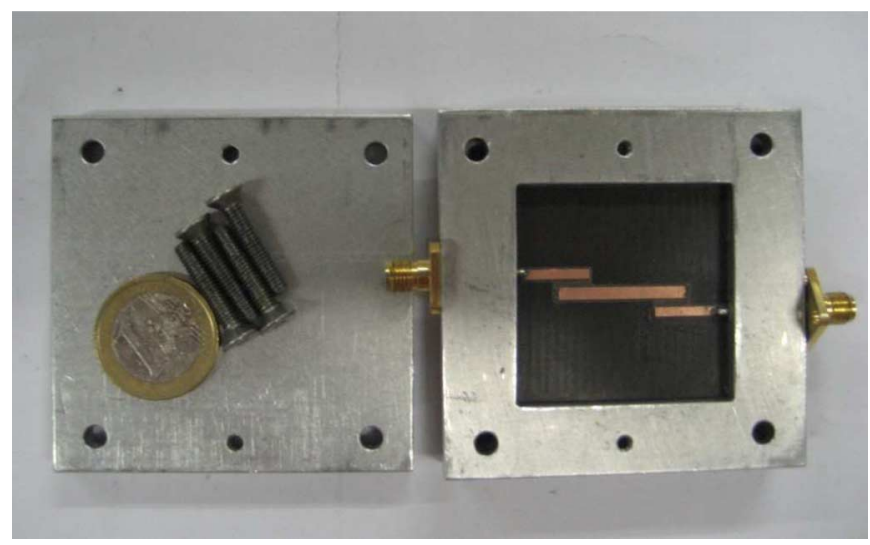

Fig. 12. Fabricated prototype showing all the pieces of the filter.

lower quality factor. In Fig. 11, we observe good agreement between measured and full-wave simulations.

\section{B. Third-Order Fully Canonical Transversal Filter Implementation}

Here we demonstrate how a third degree filtering function can be directly implemented with the compact hybrid waveguide-microstrip technology proposed. Being fully canonical, the third-order transversal structure will be able to generate up to three transmission zeros in the transfer function of the filter. This is indeed the case, as there will be a direct coupling different from zero $\left(M_{S L} \neq 0\right)$.

Nevertheless, not all the combinations in the positions of the transmission zeros can be implemented with the structure proposed. The limitation comes in the number of negative couplings that the hybrid structure, proposed in Figs. 2 and 3, can implement. As already discussed in Section II, this structure can only implement one negative coupling. This negative coupling is due to the sign change of the $x$-component of the electric field associated to the LSM mode excited in the structure. The other two resonances are formed by two half-wavelength printed line resonators and, therefore, no sign change can be implemented. Thereby the limitation is that only matrices of the form shown in Table II, which, in addition only require one negative coupling, can be implemented with the proposed topology. If two 
TABLE VII

Dimensions OF THE THIRD-DEgREE FiLTER With THREE TRANSMISSION ZEROS IN THE FREQUENCY AXIS

\begin{tabular}{c|c}
\hline Dimension & $\begin{array}{c}\text { Value } \\
\text { (in millimeters) }\end{array}$ \\
\hline \hline$a$ & 40 \\
$b$ & 41.20 \\
$L_{1}$ & 3 \\
$L_{2}$ & 1.85 \\
$L_{i n}=L_{\text {out }}$ & 14 \\
$L_{r 2}, L_{r 3}$ & $24.12,24.52$ \\
$w_{1}=w_{2}$ & 2.30 \\
$\varepsilon_{r}$ & 2 \\
\hline
\end{tabular}

negative couplings are required, then one of the printed line resonators must be modified in order to allow for the second sign change. In this case, alternative printed resonators, as described in [15], could be employed.

The first prototype that we can implement with the proposed hybrid structure is a transfer function with three transmission zeros at finite frequencies, two below the passband and one above the passband. In this example, we have placed the transmission zeros at $f_{1}=4.37 \mathrm{GHz}, f_{2}=4.51 \mathrm{GHz}$, and $f_{3}=$ $4.76 \mathrm{GHz}$. The return loss of the filter is $-18 \mathrm{~dB}$ and the center frequency is $4.7 \mathrm{GHz}$ with a bandwidth of $70 \mathrm{MHz}$. Thereby, the normalized transmission zeros result to be $s=-j \cdot 10.6311$, $s=-j \cdot 5.7546$ and $s=j \cdot 2.2097$. Following the technique presented in [3], the following $(N+2)$ by $(N+2)$ coupling matrix is obtained:
$M_{5}=\left(\begin{array}{ccccc}0 & 0.5246 & 0.4324 & -0.7571 & 0.0078 \\ 0.5246 & 1.3717 & 0 & 0 & 0.5246 \\ 0.4324 & 0 & -1.3192 & 0 & 0.4324 \\ -0.7571 & 0 & 0 & -0.1553 & 0.7571 \\ 0.0078 & 0.5246 & 0.4324 & 0.7571 & 0\end{array}\right)$.
We see that only one sign change is required in the couplings of the three resonators $\left(J_{1}=J_{2}, J_{3}=J_{4}, J_{5}=-J_{6}\right)$. The direct input/output coupling is also different from zero, as it corresponds to a fully canonical configuration. Therefore, this coupling matrix can be directly implemented with the proposed hybrid structure. The coupling matrix $M_{5}$ also indicates that the resonator changing sign must be tuned between the other two resonators $\left(M_{11}<M_{33}<M_{22}\right)$. Consequently, in the hybrid structure, we have to tune the LSM mode in between the resonances of the two printed line resonators. Taking this basic design criterion, the final dimensions of the structure, according to Figs. 2 and 3, are collected in Table VII.

The response obtained from the $M_{5}$ coupling matrix is shown in Fig. 13, where it is clear that all the specifications are met. Using the geometrical values shown in Table VII for the designed hybrid structure, the electrical behavior of the filter is obtained. This is also presented in Fig. 13, again showing very good agreement.

It is interesting to note that a filter with symmetric characteristics to the previous one (two transmission zeros above and one below the passband) cannot be obtained with the proposed hybrid structure. This is due to the fact that two sign changes must be implemented, as it can be easily verified from the coupling matrix of this new structure. However, since the design

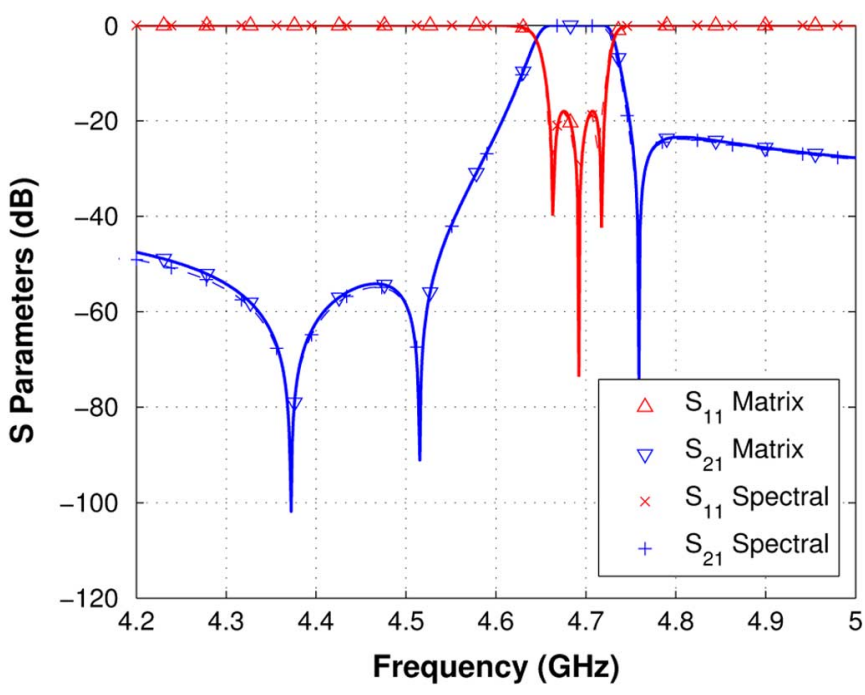

Fig. 13. Ideal model and simulated results for the bandpass transversal filter with three transmission zeros.

shown in Fig. 13 has two transmission zeros on one side of the passband, it would be relatively easy to combine them into the complex plane. Thus, that design would produce a pair of complex transmission zeros, together with one transmission zero in the frequency axis (above the passband).

\section{CONCLUSION}

In this paper, a novel hybrid structure has been proposed for the implementation of both second- and third-order filtering functions using transversal topologies. This novel structure is based on the combination of two well-known technologies: the microstrip and waveguide. In this way, couplings from the input and output ports to several resonators can be implemented in a very compact form. This paper has shown the capability of the new structure to implement a wide class of second-order fully canonical transfer functions. Furthermore, a filter with a pair of transmission zeros placed in the complex plane has been designed. This filter can improve group-delay equalization by sacrificing selectivity. In addition, a fully canonical transversal filter of order 3 is for the first time implemented using the new hybrid structure. The validity of the new proposal has been confirmed by means of several useful implementation examples, and a prototype has also been manufactured and tested with good results.

\section{REFERENCES}

[1] R. J. Cameron, "General coupling matrix synthesis methods for Chebyshev filtering functions," IEEE Trans. Microw. Theory Tech., vol. 47, no. 4, pp. 433-433, Apr. 1999.

[2] U. Rosenberg and S. Amari, "Novel coupling schemes for microwave resonator filters," IEEE Trans. Microw. Theory Tech., vol. 50, no. 12 , pp. 2896-2902, Dec. 2003.

[3] R. J. Cameron, "Advanced coupling matrix synthesis techniques for microwave filters," IEEE Trans. Microw. Theory Tech., vol. 51, no. 1, pp. 1-10, Jan. 2003.

[4] D. C. Rebenaque, A. A. Melcon, and M. Guglielmi, "A new simple microstrip open-loop resonators filter for high selectivity applications," in IEEE MTT-S Int. Microw. Symp. Dig., Philadelphia, PA, Jun. 2003 , vol. 8, no. 13, pp. 1603-1606, Paper TH-1B-3.

[5] M. Guglielmi, P. Jarry, E. Kerherve, O. Roquebrun, and D. Schmitt, "A new family of all-inductive dual-mode filters," IEEE Trans. Microw. Theory Tech., vol. 49, no. 10, pp. 1764-1769, Oct. 2001. 
[6] S. Amari and U. Rosenberg, "A universal building block for advanced modular design of microwave filters," IEEE Microw. Wireless Compon. Lett., vol. 13, no. 12, pp. 541-543, Dec. 2003.

[7] C.-K. Liao and C.-Y. Chang, "Microstrip realization of generalized Chebyshev filters with box like coupling schemes," IEEE Trans. Microw. Theory Tech., vol. 55, no. 1, pp. 147-153, Jan. 2007.

[8] S. Amari, U. Rosenberg, and J. Borneman, "Adaptive synthesis and design of resonator filters with source/load-multiresonator coupling," IEEE Trans. Microw. Theory Tech., vol. 50, no. 8, pp. 1969-1978, Aug. 2002.

[9] D. C. Rebenaque, F. Q. Pereira, J. L. G. Tornero, A. A. Melcon, and M. Guglielmi, "A new family of microstrip open-loop resonator filters for high selectivity applications," Microw. Opt. Technol. Lett., vol. 43, no. 5, pp. 450-455, Dec. 2004.

[10] M. Martínez-Mendoza, J. S. Gómez-Díaz, D. C. Rebenaque, J. L. Gómez-Tornero, and A. Alvarez-Melcon, "Design of a bandpass transversal filter employing a novel hybrid printed structure," in IEEE MTT-S Int. Microw. Symp. Dig., Honolulu, HI, Jun. 2007, vol. 6, pp. 1281-1284.

[11] R. E. Collin, Field Theory of Guided Waves. Piscataway, NJ: IEEE Press, 1991

[12] M. Guglielmi, "A simple CAD procedure for microwave filters and multiplexers," IEEE Trans. Microw. Theory Tech., vol. 42, no. 7, pp. 1347-1352, Jul. 1994.

[13] D. Swanson and G. Macchiarella, "Microwave filter design by synthesis and optimization," IEEE Micro, pp. 55-69, Apr. 2007.

[14] A. A. Melcon, J. R. Mosig, and M. Guglielmi, "Efficient CAD of boxed microwave circuits based on arbitrary rectangular elements," IEEE Trans. Microw. Theory Tech., vol. 47, no. 7, pp. 1045-1058, Jul. 1999.

[15] D. C. Rebenaque, F. Q. Pereira, J. P. Garcia, A. A. Melcon, and M. Guglielmi, "Two compact configurations for implementing transmission zeros in microstrip filters," IEEE Microw. Wireless Compon. Lett., vol. 14, no. 10, pp. 475-477, Oct. 2004.

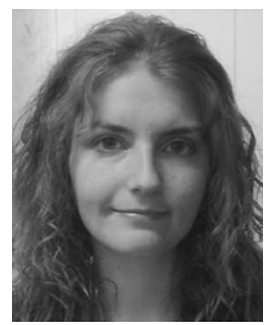

Mónica Martinez-Mendoza was born in Cartagena, Murcia, Spain, in 1983. She received the Telecommunications Engineer degree from the Technical University of Cartagena (UPCT), Cartagena, Spain, in 2006, and is currently working toward the Ph.D. degree at UPCT.

In 2007, she joined the Telecommunications and Electromagnetic Group, UPCT, as a Research Assistant, where she is involved in the development of novel transversal filtering structures for satellite systems. Her current scientific interests include the analysis and design of microwave circuits.

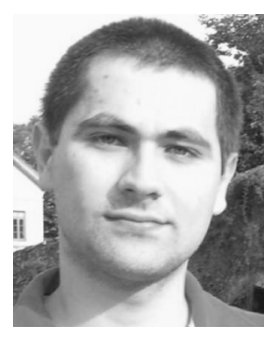

Juan Sebastián Gómez-Díaz was born in Albacete, Spain, in 1983. He received the Telecommunications Engineer degree (with honors) from the Technical University of Cartagena (UPCT), Cartagena, Spain, in 2006, and is currently working toward the Ph.D. degree at UPCT.

In 2007 he has joined the Telecommunication and Electromagnetic Group, UPCT, as a Research Assistant. His current scientific interests include numerical methods and their application in the analysis and design of microwave circuits and antennas.

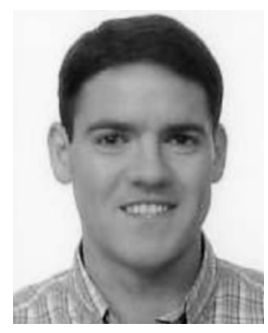

David Cañete-Rebenaque ( $\mathrm{S}^{\prime} 04$ ) was born in Valencia, Spain, in 1976. He received the Telecommunications Engineer degree from the Technical University of Valencia, Valencia, Spain, in 2000, and is currently working toward the Ph.D. degree at the University of Cartagena, Cartagena, Spain.

During 2001, he was an RF Engineer with a mobile communication company. In 2002, he joined the Communications and Information Technologies Department, Technical University of Cartagena (UPCT). His research interests include analysis and design of microwave circuits and active antennas.

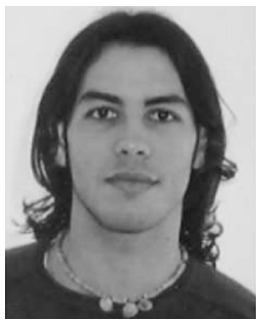

Jose Luis Gómez-Tornero (M'06) was born in Murcia, Spain, in 1977. He received the Telecommunications Engineer degree from the Universidad Politécnica de Valencia (UPV), Valencia, Spain, in 2001, and the Ph.D. degree (laurea cum laude) in telecommunication engineering from the Technical University of Cartagena (UPCT), Cartagena, Spain, in 2005.

In 1999, he joined the Radiocommunications Department, UPV, as a Research Student, where he was involved in the development of analytical and numerical tools for the study and automated design of microwave filters in waveguide technology for space applications. In 2000, he joined the Radio Frequency Division, Industry Alcatel Espacio, Madrid, Spain, where he was involved with the development of microwave active circuits for telemetry, tracking, and control (TTC) transponders implicated in many different spatial missions for the European Space Agency (ESA), National Aeronautics Space Administration (NASA), and other space agencies. In 2001, he joined the UPCT, as an Assistant Professor, where he currently develops his teaching activities. Since October 2005, he has been Vice Dean for students and lecture affairs with the Telecommunication Engineering Faculty, UPCT. His scientific research is focused on the analysis and design of leaky-wave antennas for millimeter-waveband applications and the development of numerical methods for the analysis of novel passive radiating structures in planar and waveguide technologies. His scientific interests also include the study of active devices for microwave and millimeter wavebands such as oscillators and active antennas.

Dr. Gómez-Tornero was the recipient of the 2004 Second National Award presented by the EPSON-Ibérica Foundation for the best doctoral project in the field of technology of information and communications (TIC). He was also the recipient of the 2006 Vodafone Foundation Colegio Oficial de Ingenieros de Telecomunicación (COIT/AEIT) Award presented to the best Spanish doctoral thesis in the area of advanced mobile communications technologies.

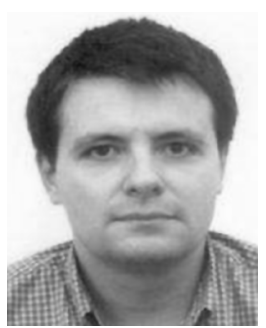

Alejandro Alvarez-Melcon (M'99-SM'07) was born in Madrid, Spain, in 1965. He received the Telecommunications Engineer degree from the Technical University of Madrid (UPM), Madrid, Spain, in 1991, and the Ph.D. degree in electrical engineering from the Swiss Federal Institute of Technology, Lausanne, Switzerland, in 1998.

In 1988, he joined the Signal, Systems and Radiocommunications Department, UPM, as a Research Student, where he was involved in the design, testing, and measurement of broadband spiral antennas for electromagnetic measurements support (EMS) equipment. From 1991 to 1993, he was with the Radio Frequency Systems Division, European Space Agency (ESA)/European Space Research and Technology Centre (ESTEC), Noordwijk, The Netherlands, where he was involved in the development of analytical and numerical tools for the study of waveguide discontinuities, planar transmission lines, and microwave filters. From 1993 to 1995, he was with the Space Division, Industry Alcatel Espacio, Madrid, Spain, and also with the ESA, where he collaborated on several ESA/ESTEC contracts. From 1995 to 1999, he was with the Swiss Federal Institute of Technology, École Polytechnique Fédérale de Lausanne (EPFL), Lausanne, Switzerland, where he was involved in the field of microstrip antennas and printed circuits for space applications. In 2000, he joined the Technical University of Cartagena (UPCT), Cartagena, Spain, where he currently develops his teaching and research activities.

Dr. Alvarez-Melcón was the recipient of the Journée Internationales de Nice Sur les Antennes (JINA) Best Paper Award for the best contribution to the JINA'98 International Symposium on Antennas, and the Colegio Oficial de Ingenieros de Telecomunicación (COIT/AEIT) Award for the best doctoral thesis in basic information and communication technologies. 